TecnoLógicas

ISSN-p 0123-7799

ISSN-e 2256-5337

Vol. 22, No. 45 , pp. $45-58$

Mayo-agosto de 2019

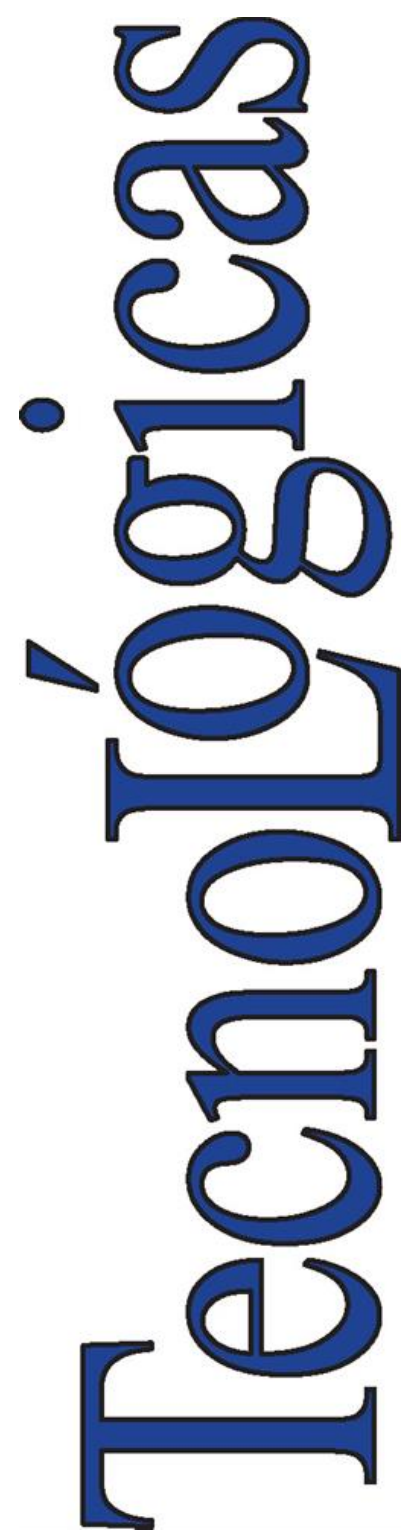

(C) Instituto Tecnológico Metropolitano Este trabajo está licenciado bajo una Licencia Internacional Creative

Commons Atribución (CC BY-NC-SA)

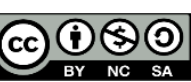

Artículo de Investigación/Research Article

\title{
Algoritmo adaptativo para protecciones de sobrecorriente en el caso de estudio IEEE9
}

\section{An adaptive algorithm for overcurrent protections on the case study IEEE9}

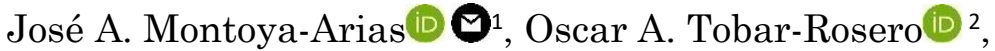 \\ German D. Zapata-Madrigal(D) ${ }^{3}$, Rodolfo García-Sierra (iD 4
}

Recibido: 15 de agosto de 2018

Aceptado: 05 de marzo de 2019

Cómo citar / How to cite

J. A. Montoya-Arias, O. A. Tobar-Rosero, G. D. Zapata-Madrigal, R. García-Sierra, "Algoritmo adaptativo para protecciones de sobrecorriente en el caso de estudio IEEE9," TecnoLógicas, vol. 22, no. 45 , pp. $45-58,2019$. https://doi.org/10.22430/22565337.1335

1 Ingeniero Electricista, Universidad Nacional de Colombia Sede Medellín, Medellín-Colombia, joamontoyaar@unal.edu.co

2 Ingeniero Electricista, Universidad Nacional de Colombia Sede Medellín, Medellín-Colombia, oatobarr@unal.edu.co

$3 \mathrm{PhD}$. en ingeniería, Ingeniero Electricista, Profesor asociado de la Universidad Nacional de Colombia Sede Medellín, Medellín-Colombia, gdzapata@unal.edu.co

4 PhD. en ingeniería, Ingeniero Electricista, CODENSA grupo Enel, MedellínColombia, rodolfo.garcia@enel.com 


\section{Resumen}

Una adecuada coordinación de protecciones eléctricas es fundamental en los sistemas de potencia, con el fin de evitar desconexiones inesperadas que pueden desencadenar problemas en la operación de la red eléctrica y sus diferentes componentes. Aunque esta actividad tiene una alta complejidad en sistemas de gran tamaño, actualmente existen algunos métodos de coordinación de protecciones automáticos, los cuales han sido aplicados en casos de estudio. En este documento se desarrolla un algoritmo adaptativo de coordinación de cuatro relés de sobrecorriente direccionales, que se encuentran en dos líneas de transmisión adyacentes en el caso de estudio de nueve barras IEEE 9; dos protecciones por cada línea de transmisión seleccionada. Posteriormente se encuentra un análisis de los resultados obtenidos, explicando ciertas características del sistema de potencia y relacionándolas con el comportamiento de los parámetros de los relés ante los cambios en la topología de este. Finalmente, se mencionan las conclusiones sobre dichos resultados y el desempeño del algoritmo. Los aportes de este trabajo radican en el análisis de una alternativa innovadora en Colombia, que permita optimizar el uso del esquema de protecciones en un sistema de potencia. Asimismo, los resultados obtenidos permiten validar la implementación del algoritmo en equipos reales, para un piloto sobre un segmento de la red, el cual es propiedad de CODENSA.

\section{Palabras clave}

Protecciones eléctricas, Coordinación de protecciones, Algoritmo adaptativo, Sistemas de potencia, Topología de red.

\section{Abstract}

A proper coordination of electrical protections is fundamental in electric power systems, with the aim of avoiding unexpected disconnections. These disconnections can produce problems in the operation of the power system and in its different components. Even though this activity has complex tasks in great power systems, currently exist some automatic methods for coordination of protections applicated in study cases. In this document, an adaptive algorithm for coordination of four directional overcurrent relays located in two adjacent transmission lines in the nine bars study case IEEE9 is developed. Two protections per transmission line. Afterwards, an analysis of the obtained results is exhibited, explaining some characteristics of the power system and relating them with the behavior of the parameters of the relays, due to topology changes in the network. Finally, the conclusions about the results and the performance of the algorithm are mentioned. The contributions of this work are shown in the analysis of an innovative alternative in Colombia, that allows optimize the use of the protections scheme in power systems. Likewise, the obtained results allow to validate the implementation of the algorithm in real devices in a segment of the electric network in Colombia, which is property of CODENSA.

\section{Keywords}

Electric protections, Protections coordination, Adaptive algorithm, Power systems, Network Topology. 


\section{INTRODUCCIÓN}

En los sistemas eléctricos de potencia se emplean típicamente relés de distancia y relés direccionales de sobrecorriente como protecciones para las líneas de transmisión y subtransmisión. El cálculo de los parámetros de cada relé del sistema debe hacerse de manera cuidadosa debido a que la curva de protección de cada uno puede solaparse con la del relé adyacente y ocasionar problemas de selectividad; es decir que, no existe algún cruce entre ellas, permitiendo a los relés operar en orden y sin presentar accionamientos simultáneos.

Las protecciones direccionales de sobre corriente se pueden coordinar de dos formas diferentes: convencional o manual y basados en métodos de optimización [1], [2]. La forma manual se divide en: Ensayo - error, y análisis de topologías. Estos métodos son fáciles de implementar cuando la red eléctrica es pequeña; no así, cando la red es robusta y cuenta con varias mallas. Por lo anterior, típicamente son utilizados en circuitos radiales, pero cuando la topología es más compleja o existen muchas líneas, se vuelve una tarea algo más tediosa.

Actualmente, en Colombia se realiza el ajuste de protecciones alternando dichos métodos, más no se cuenta con experiencias documentadas sobre la aplicación de métodos de optimización o coordinación de protecciones eléctricas, con algoritmos adaptativos; lo cual denota que, existe desconocimiento sobre el tema 0 desconfianza en su implementación.

Los métodos de optimización se han convertido en herramientas muy prácticas, escogidas por investigadores para la coordinación de protecciones de grandes sistemas de potencia; de los cuales, varios se han utilizado en la coordinación real de redes [3]-[8]. Estos métodos de optimización definen funciones objetivo basadas en el tipo de protecciones a coordinar o esquema de protección, con combinaciones de varios tipos de relés
[7],[9]-[11]. Dichas funciones presentan no linealidades, que no se pueden resolver a mano si el sistema es de gran dimensión; y adicional a esto, cada protección va a tener varios tipos de restricciones dependiendo del número de respaldos que posea [6].

Una protección adaptativa se puede entender como un relé que tiene la capacidad de alterar o cambiar sus parámetros de operación en cualquier momento, ya sea por decisión propia, donde el algoritmo esté programado en él; o por orden de un equipo externo, con el fin de adecuarse a un cambio de topología que se presente en el sistema de potencia [5],[12][16].

En este documento se presenta un método para la coordinación adaptativa de cuatro protecciones de sobrecorriente, utilizando el método manual, basado en el análisis de topologías programado mediante Python.

El proceso de pruebas aplicado permitirá observar las ventajas que tiene la implementación de un algoritmo adaptativo en la coordinación de protecciones y a su vez, aporta a que se lleve a cabo una mayor exploración del tema, para su implementación real en el sistema eléctrico colombiano.

Este trabajo presenta un método adaptativo para la coordinación de cuatro protecciones direccionales de sobrecorriente en la sección 2, el cual explica los pasos a seguir para el cálculo de los parámetros de los relés en el caso de estudio. En la sección 3 se encuentra la definición del algoritmo adaptativo implementado y el proceso que este comprende, para luego de su ejecución presentar un análisis de los resultados obtenidos en la sección 4, y finalizar con las conclusiones obtenidas en el proceso.

\section{COORDINACIÓN DE PROTECCIONES DEL CASO DE ESTUDIO}

La coordinación de protecciones direccionales de sobrecorriente en 
sistemas de potencia debe cumplir con un criterio de selectividad entre ellas. Esto significa, que los relés no deben operar cuando existan contingencias fuera de su área de actuación; es decir, cada relé tiene un rango de magnitud de corrientes definidas en las que debe operar en cierto instante de tiempo, y son esquematizados en curvas de tiempo inverso de actuación. Los cuatro relés se definen como direccionales, razón por la cual solo operan en un sentido, dependiendo de su ubicación.

El algoritmo de coordinación se emplea en el caso de estudio de nueve barras IEEE 9 (ver Fig. 1), el cual típicamente se utiliza para el análisis de sistemas de potencia. Este sistema cuenta con 3 generadores sincrónicos, 3 transformadores trifásicos y 6 líneas de transmisión de $230 \mathrm{kV}$. Los parámetros de las líneas del sistema de potencia se presentan en la Tabla 1

$\mathrm{Al}$ observar la Fig. 1, las líneas de transmisión donde están los relés de sobrecorriente direccionales son, la Línea 89 y la Línea 94, comprendidas entre las barras B8, B9 y B4. La coordinación de estas protecciones se realiza teniendo en cuenta la corriente que monitorea cada relé al suceder una falla trifásica en la barra
Tabla 1. Parámetros de las líneas de transmisión en caso de estudio IEEE 9. Fuente: autores.

\begin{tabular}{cccc}
\multicolumn{4}{c}{ de estudio IEEE 9. Fuente: autores. } \\
\hline Desde & Hasta & $\mathrm{R}(\Omega)$ & $\mathrm{X}(\Omega)$ \\
\hline 1 & 4 & 0 & 0.0576 \\
3 & 8 & 0 & 0.0625 \\
4 & 6 & 0 & 0.0586 \\
5 & 5 & 0.0101 & 0.0850 \\
6 & 6 & 0.0320 & 0.1610 \\
7 & 7 & 0.0170 & 0.0920 \\
8 & 8 & 0.0085 & 0.0720 \\
9 & 9 & 0.0119 & 0.1004 \\
\hline
\end{tabular}

adyacente de cada uno. Por esto, los relés S1 y S4 se calculan con la corriente de corto que detectan al hacer una falla trifásica en la barra 9, el relé S2 con falla en la barra 4 y el relé $\mathrm{S} 3$ con falla en la barra 8 .

Se puede apreciar que $\mathrm{S} 1$ se debe coordinar como respaldo de S3, al igual que S4 es el respaldo de S2. La forma manual de coordinación en la que se basa el algoritmo se explica a continuación tomando como referencia la coordinación de S2 y S4.

-Simulación de flujos de potencia con el fin de observar el comportamiento de las

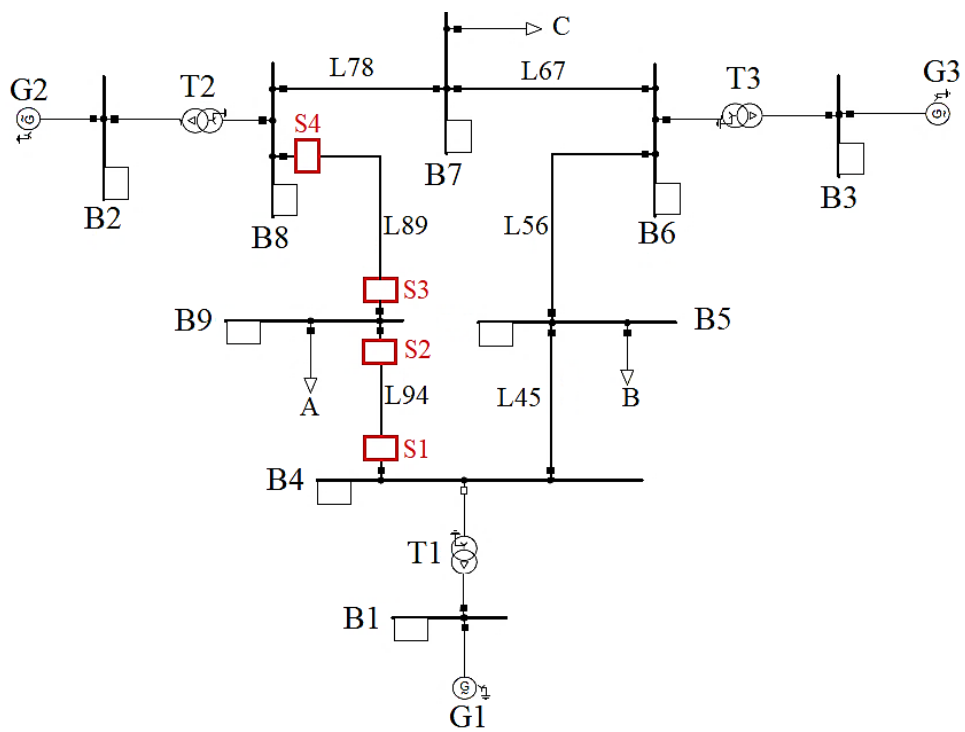

Fig. 1. Diagrama unifilar del caso de estudio IEEE9 con los relés propuestos. Fuente: autores. 
corrientes vistas por los relés ante cambios de topología del sistema (salida de líneas o de generadores), y las corrientes de mayor magnitud leídas por cada relé, se multiplican por un factor de 1.1 y se utilizan como corrientes de arranque o Ipickup de la etapa temporizada.

-Simulación de fallas trifásicas en las barras 4 y 9 para conocer la corriente que percibe cada relé.

-Asignar el mínimo TSM (Time Setting Multiplier) o Dial al relé principal, es decir, S2 y S3. Se asigna el valor de 0.05 , el cual es el valor mínimo del Dial para definir el punto de partida del esquema de protecciones con respaldo.

-Cálculo del tiempo de actuación del relé, con base a la corriente vista en el momento de la falla trifásica en la barra aguas abajo. Para tal ejercicio se escogió el tipo de relé IEC de tiempo inverso normal (ver Fig. 2), cuya curva de actuación es regida por (1):

$$
t_{o p}=\frac{0.14}{\left(\frac{I c c_{F i}}{I_{P}}\right)^{0.02}-1} T S M_{i}
$$

Donde:

$t_{o p} \quad$ Tiempo de operación del relé

Corriente de corto vista por el

$I c c_{F i}$ relé ante la falla $\mathrm{Fi}$, donde i es el punto de falla.

$I_{P}$ : Corriente de arranque o pickup

\section{TSM del relé}

Time Setting Multiplier o Dial

-Calcular el tiempo de operación del relé de respaldo al cual se le adiciona un margen de tiempo o CTI (Coordination Time Interval). Este, por lo general es un valor de 0.2 segundos [5].

-Hallar el TSM del relé de respaldo.
Los valores de los TSM son redondeados a valores con dos decimales, debido a que los relés sólo aceptan valores con esta característica (principalmente los electromecánicos). Hoy en día el procesador de los relés acepta valores más precisos, pero se procuró tener una posición un poco conservadora en este aspecto [11].

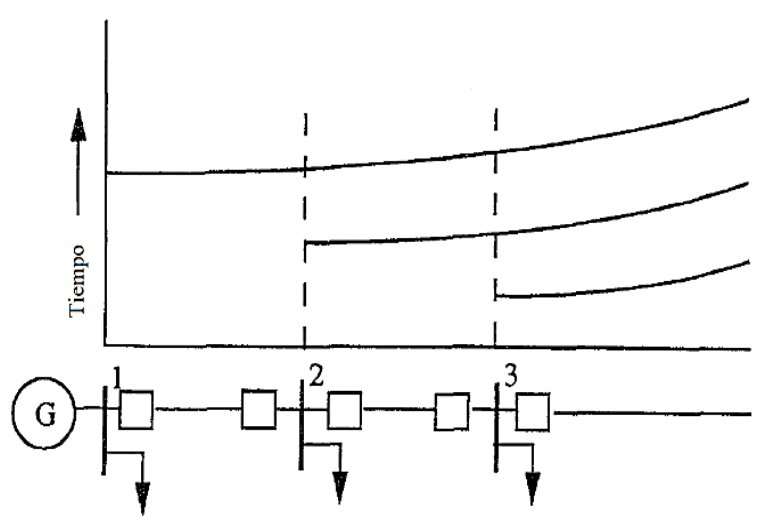

Fig. 2.Curvas de actuación de protecciones de Sobrecorriente. Fuente: [11].

\section{ALGORITMO ADAPTATIVO DE COORDINACIÓN DE PROTECIONES}

El esquema adaptativo del algoritmo se basa principalmente en el monitoreo constante del estado de los interruptores de cada línea de transmisión y de los generadores [5]. Cuando se vea afectado el estado del interruptor de algún elemento del sistema ya sea porque se presente alguna contingencia $o$ la salida programada del mismo por mantenimiento, el algoritmo es capaz de notar esta ausencia.

Lo anterior debido a que, si este está en su operación normal, el interruptor permanece cerrado permitiendo el flujo de corriente; pero si el elemento es aislado, el interruptor se abre, e inicia automáticamente el cálculo de parámetros de cada relé para la nueva topología. Este procedimiento se muestra mediante un diagrama de flujo en la Fig. 3.

El algoritmo de coordinación descrito en la sección dos, y la lógica asociada al 
algoritmo adaptativo descrita anteriormente, fue programada mediante Python, bajo los parámetros y funciones requeridos por el programa DigSilent, en el cual, se ha implementado en el caso de estudio IEEE9.

Los valores de los parámetros calculados por el algoritmo de cada relé bajo los cambios de topología en el sistema de potencia se aprecian en la Tabla 2.

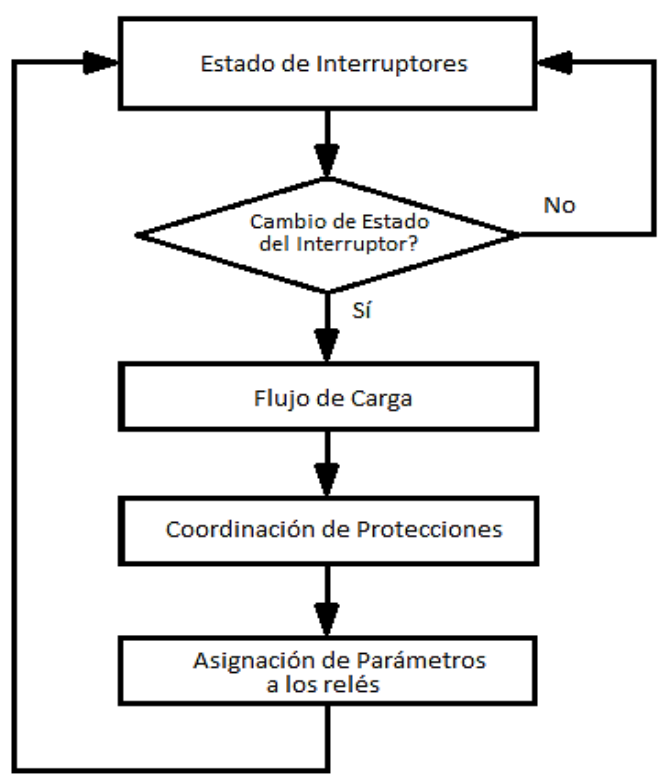

Fig. 3. Diagrama de flujo del algoritmo adaptativo. Fuente: [4].

\section{ANÁLISIS DE RESULTADOS}

En la parte inferior de la Tabla 2, cuando se analiza el algoritmo sin la línea de transmisión 89 , solo es posible coordinar el relé $\mathrm{S} 1$, debido a que en la línea que se encuentra fuera de servicio, están los relés S3 y S4, y el relé S2 no es necesario habilitarlo, ya que no existe la posibilidad de que se produzca una corriente desde la barra 9 a la barra 4 que ocasione la operación del relé.

La carga A es una carga PQ balanceada (al igual que las cargas $\mathrm{B}$ y C), y esta no hace un aporte a la corriente de corto cuando existe una falla en la línea 94 o en la barra 4, por lo que tampoco es posible calcular los parámetros de S2. La misma situación se evidencia cuando la línea 94 es desconectada, por tanto, solo es posible calcular los parámetros del relé $\mathrm{S} 4$.

En la Fig. 4, se exponen las curvas inversas de los relés de sobrecorriente en su estado de operación inicial, es decir, sin cambios en la topología del sistema. Las cargas del sistema cuando son desconectadas no alteran las impedancias equivalentes vistas por los relés, por lo que un cambio de operación de las mismas no afecta los niveles de corto del sistema.

El aumento de demanda o la salida de cargas ocasionan un cambio en la corriente de operación de las líneas, por esta razón los parámetros calculados de los relés no se afectan. Además, al hacer un análisis de contingencias $\mathrm{N}-1$, se conoce la corriente mayor a la de operación que puede circular por cada línea

Este tipo de contingencias tienen una alta probabilidad de ocurrencia y conllevan un incremento considerable de corriente a través de las líneas de transmisión, la cual depende de la topología

Conociendo estas magnitudes, se ajustan las corrientes de arranque de los relés de sobrecorriente. Como se observa en la Tabla 2, las corrientes de arranque o pickup de todos los relés se han dejado fijas, independiente del cambio de topología que sufra el sistema.

Estas corrientes de arranque han ocasionado cierta dificultad a la hora de coordinar los relés S3 y S1, debido a que la corriente de arranque de $\mathrm{S} 1$ es menor que la corriente de arranque de S3. Esto causaría un cruce entre las curvas inversas de estos relés en la etapa temporizada.

Para evitar dicho solapamiento, hay que incrementar el Dial de S1 (aumentando su tiempo de actuación) o fijar una corriente mayor a la de S3. Es por esto que se define un nuevo valor de corriente de arranque, el cual consiste en cambiar el valor de esta corriente calculada entre S1 y S3; en consecuencia, el valor de S3 es asignado a S1 y viceversa. 
Al implementar este cambio, el Dial de S1 pasa de ser 0.18 a ser 0.15 , el cual se observa en la Tabla 2 cuando la topología está completa.

Otra condición crítica de corrientes de operación que puede suceder en la línea 94, es bajo una contingencia N-2 en la que el generador 2 y la línea 45 están fuera de servicio. En dicho caso, la corriente de operación vista por $\mathrm{S} 1$ es de $690 \mathrm{~A}$, la cual podría hacer operar el relé $\mathrm{S} 1$, confundiéndolo con una corriente que aporta a una falla ocasionando la apertura de la línea, debido a que esta corriente se encuentra dentro del rango temporizado. La probabilidad de ocurrencia de este tipo de contingencias es muy baja, por lo que se considera que rara vez se presentará.

Si las corrientes de arranque manejan una proporción con la corriente de operación, dependiendo de la topología, se

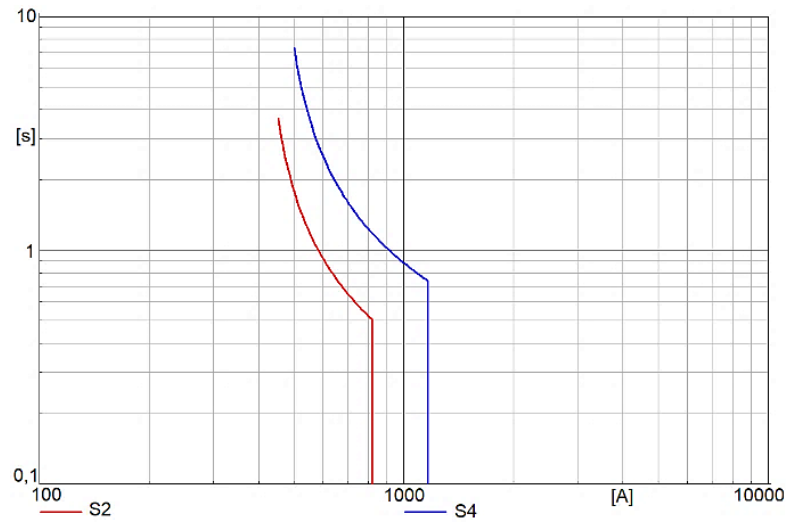

pueden presentar valores muy bajos, y causar cruce de curvas de protecciones. Un ejemplo de esto es $\mathrm{S} 2$ y $\mathrm{S} 4$ cuando el generador 3 está fuera de servicio, y las corrientes de arranque de los relés se configuran como la corriente de operación de la línea multiplicada por un factor de 1.5, como se aprecia en la Fig. 5.

A continuación, se hace una comparación de la operación de los relés cuando poseen los parámetros calculados con la coordinación original y ocurre un cambio de topología en el sistema de potencia.

En la Fig. 6 se muestra el caso en que la línea 45 está fuera de servicio, y ocurrió una falla al 50\% de la línea 89. En la Fig. 6a, los parámetros de los relés conservan los valores calculados inicialmente, cuando el sistema no tiene cambio de topología.

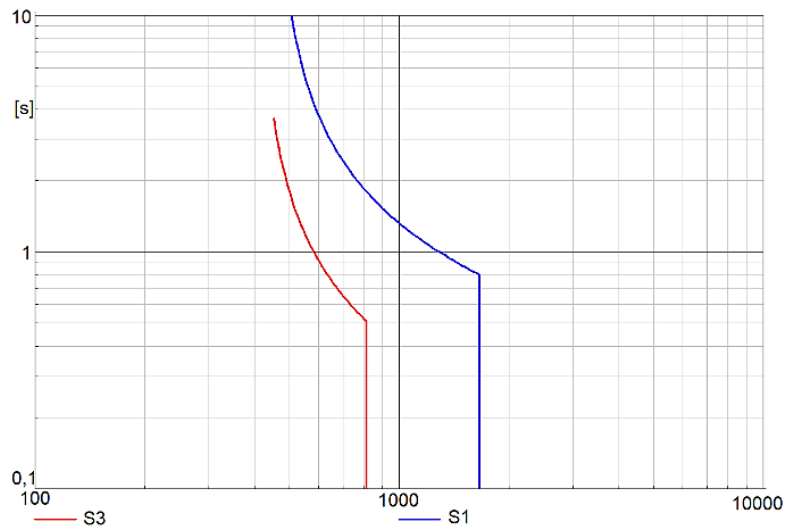

Fig. 4. Curvas de los relés sin cambio de topología en el sistema de potencia. a) S2 y S4 b) S3 y S1. Fuente: autores.

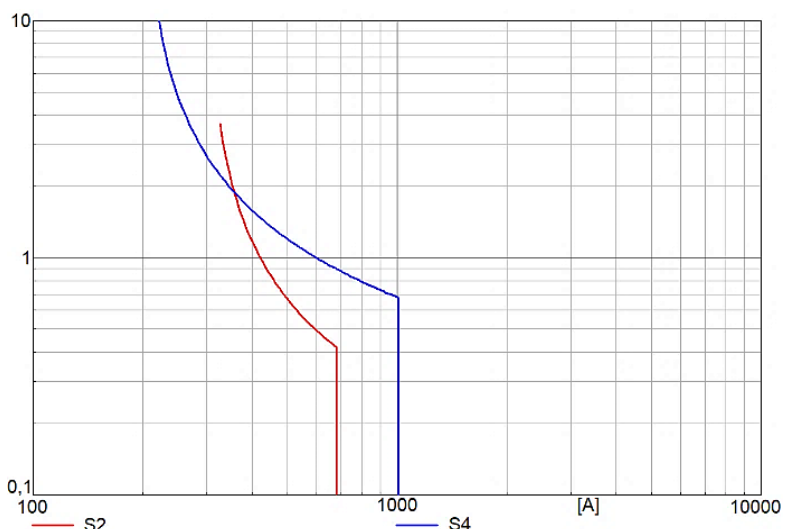

Fig. 5. Solapamiento de curvas de actuación entre S2 (rojo) y S4 (azul). Fuente: autores. 


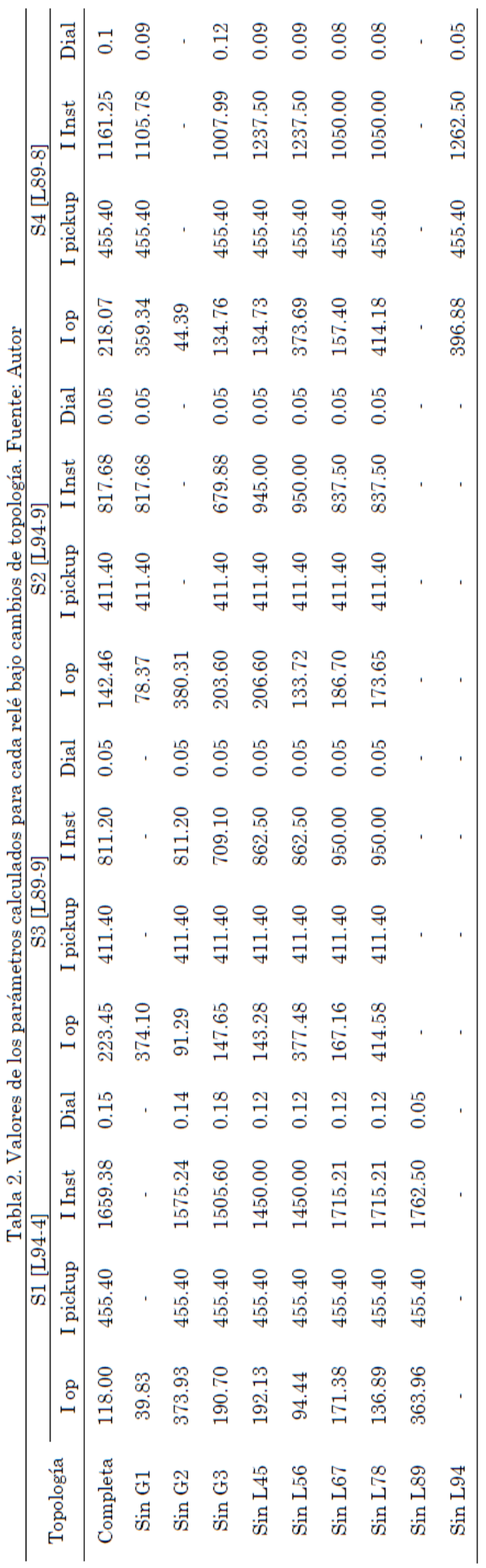

Esta gráfica indica que el relé S3 actúa instantáneamente cuando se presenta la falla, y S1 actuaría en 1.626 segundos si S3 no lo hace, operando como relé de respaldo. La Fig. 6b muestra las mismas condiciones operativas en cuanto a lugar y tipo de falla.

El tiempo de respuesta de S1 ahora disminuyó a 1.301 segundos debido a que el Dial de este pasó de ser de 0.15 a 0.12. También hubo un aumento en el nivel del corto del sistema visto por S3 debido a la ausencia de dicha línea, lo que aumenta la corriente instantánea del mismo, pero sigue operando de forma inmediata.

En la Fig. 7 se expone el mismo caso anterior, pero ahora para los relés S2 y S4. Como estos son direccionales y la condición de falla conduce el flujo de potencia en dirección contraria a la dirección de protección de los equipos, únicamente ven las corrientes, pero no actúan. De igual manera se puede ver el cambio de parámetros en los mismos, reflejados en las curvas inversas, mostrando el incremento en el nivel de corto del sistema y en el aumento de las corrientes instantáneas de ambas protecciones, adicional a una disminución en el Dial de $\mathrm{S} 4$.

En la Fig. 8 se muestra la misma comparación del cambio de topología al encontrarse deshabilitada la línea 45 , pero esta vez se presenta la falla trifásica al $50 \%$ de la línea 94. La Fig. 8a muestra los relés $\mathrm{S} 1$ y S3 con la coordinación inicial, la cual fue llevada a cabo con el sistema completo sin cambio de topología, y su comportamiento ante la falla y la ausencia de la línea 45.

En esta situación, S3 no opera debido a la dirección de la corriente de línea presente ante la falla, la cual es contraria a la direccionalidad programada en el equipo, y S1 opera en 0.915 segundos. En la Fig. 8b, cuando el algoritmo de coordinación calcula los nuevos parámetros referentes a la nueva topología, se aprecia nuevamente el incremento de la corriente instantánea en S3 como en el caso anterior 
con la falla en la línea 89 , pero no operaría por la misma condición anterior.

No obstante, en S1 la corriente instantánea disminuye ligeramente, al igual que su Dial, presentando un tiempo de actuación de 0.732 segundos, cerca de la etapa instantánea. Este tiempo de actuación es ligeramente menor al presentado en el caso donde no se lleva a cabo la aplicación del algoritmo.

Las curvas inversas de los relés $\mathrm{S} 2$ y $\mathrm{S} 4$ de la Fig. 9, expresan el comportamiento de estos dos relés ante la presencia de una falla trifásica en el 50\% de la línea 94. En la Fig. 9a se aprecian las curvas de los relés con los parámetros calculados en la coordinación inicial con todos los elementos del sistema de potencia en operación normal.
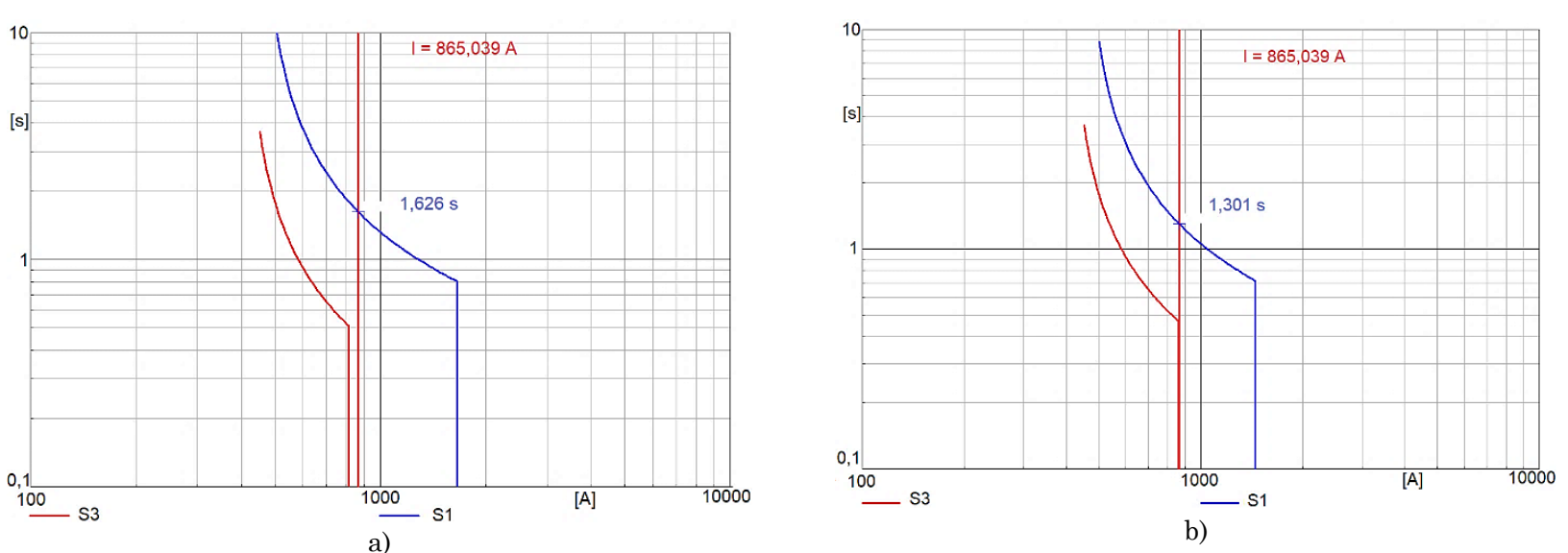

b)

El punto de falla al estar tan cerca de S2, hace operar este relé de manera instantánea. En el caso de S4, al percibir la corriente que pasa por la línea ante la falla, tarda 1.10 segundos antes de operar, operando como respaldo de S2. En la Fig. $9 \mathrm{~b}$, se expone cómo el algoritmo adaptativo coordina las protecciones teniendo en cuenta la nueva topología.

Como se mencionó anteriormente, el nivel de corto del sistema de potencia aumentó, y se ve reflejado en el incremento de las corrientes instantáneas de ambos relés. S2 ya no opera de manera instantánea, tardando en esta ocasión 0.474 segundos, y el tiempo de actuación de S4 disminuyó quedando en 0.990 segundos.

de S3 (rojo) y S1 (azul), sistema de potencia sin la línea 45 y ante una falla en la línea 89 al 50\%. a) Coordinación inicial. b) Coordinación adaptativa. Fuente: autores.
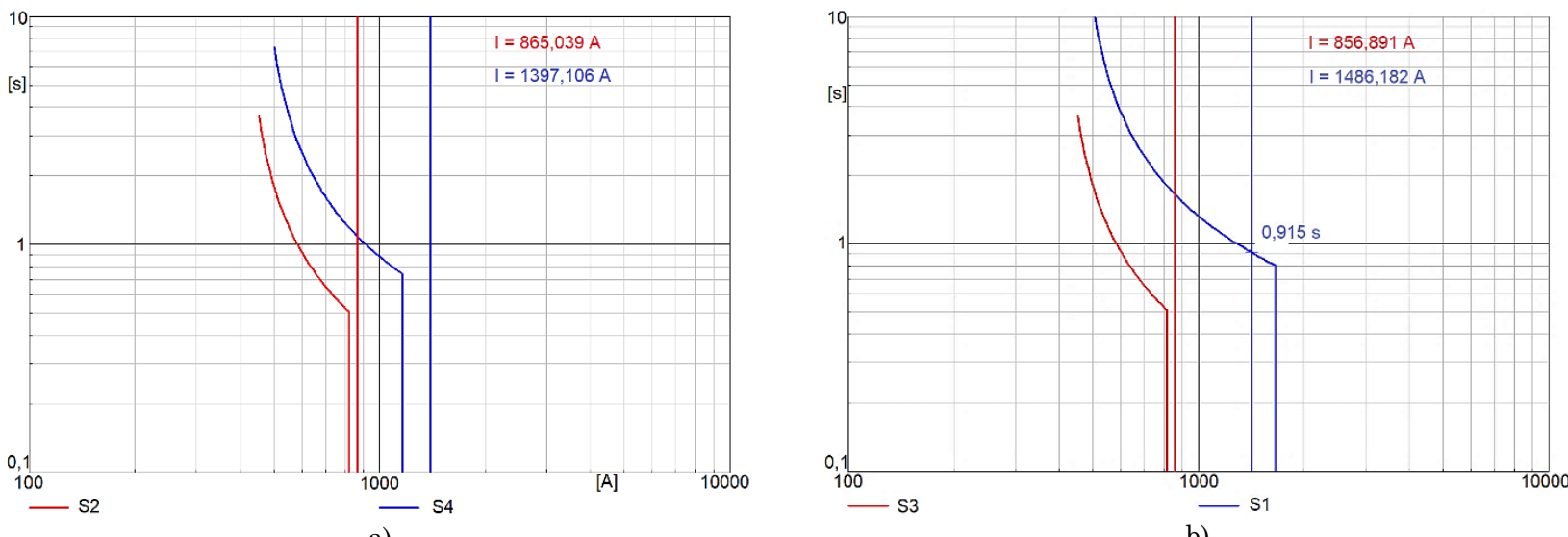

a)

Fig. 7. Curvas de operación de S2 (rojo) y S4 (azul), sistema de potencia sin la línea 45 y ante una falla en la línea 89 al $50 \%$. a) Coordinación inicial. b) Coordinación adaptativa. Fuente: autores. 

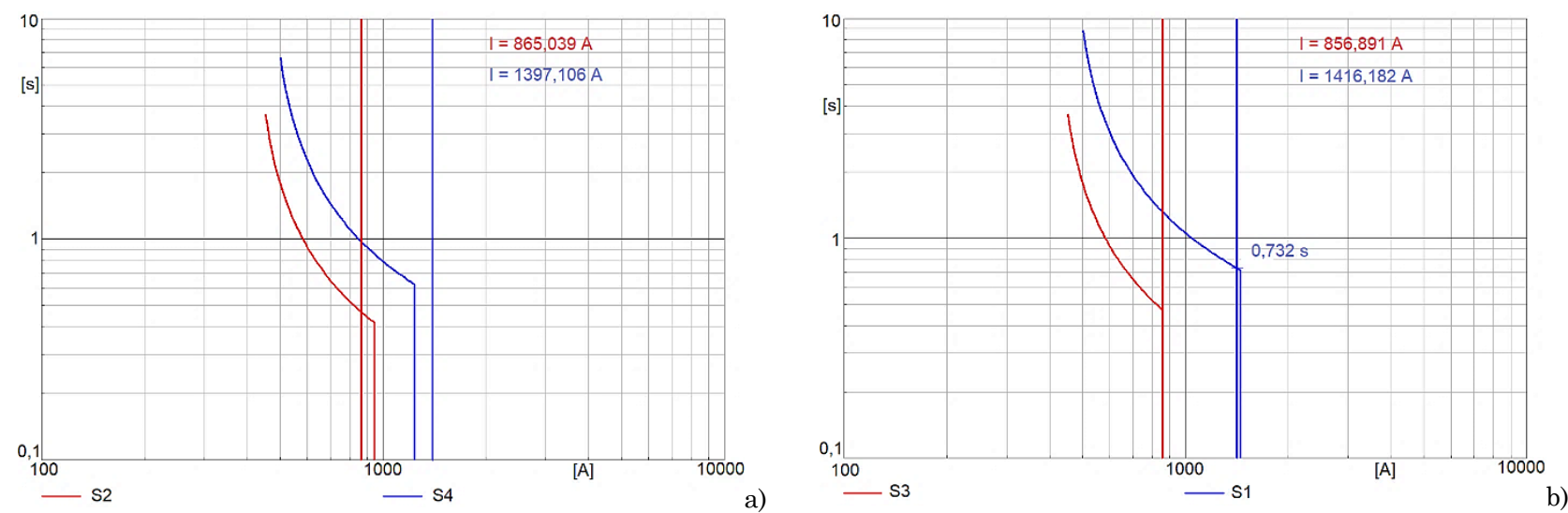

Fig. 8. Curvas de operación de S3 (rojo) y S1 (azul), sistema de potencia sin la línea 45 y ante una falla en la línea 94 al $50 \%$. a) Coordinación inicial. b) Coordinación adaptativa. Fuente: autores.

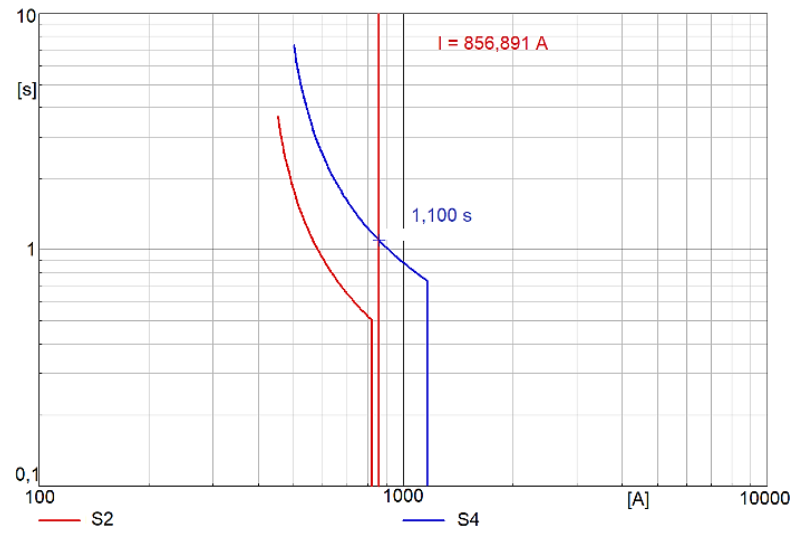

a)

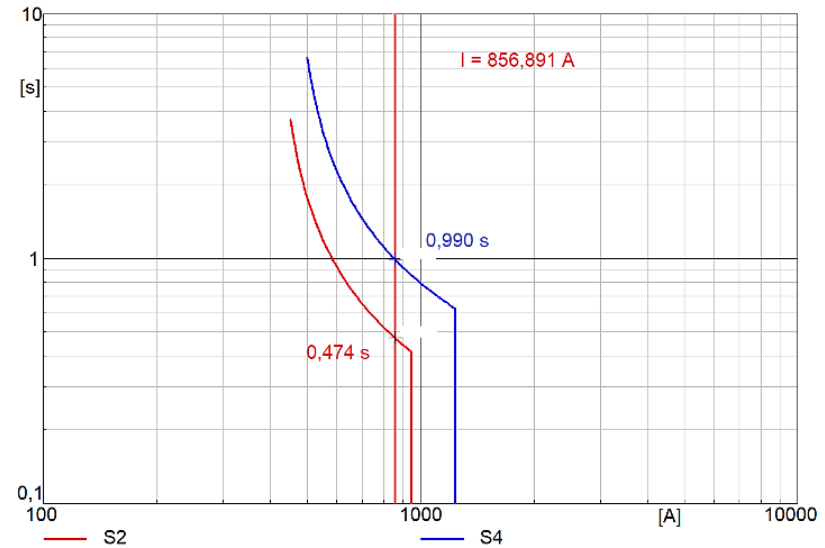

b)

Fig. 9. Curvas de operación de S2 (rojo) y S4 (azul), sistema de potencia sin la línea 45 y ante una falla en la línea 94 al $50 \%$. a) Coordinación inicial. b) Coordinación adaptativa. Fuente: autores.

Los resultados de la coordinación adaptativa del sistema de potencia comparados con los resultados obtenidos con el cambio de topologías y la coordinación inicial se detallan en la Tabla 3, donde se puede apreciar una disminución de los tiempos de actuación en la mayoría de los casos cuando se implementa el algoritmo adaptativo de coordinación.

Cuando ocurre la salida del generador 1 , no es posible calcular los parámetros de $\mathrm{S} 3$ ni de S1, debido a que la corriente vista por S3 ante la respectiva falla trifásica realizada para su coordinación (202.22 A), es menor a la corriente de arranque asignada al mismo (411.4 A) y a la corriente nominal de la línea bajo esta contingencia (374.096 A), dando como resultado un tiempo de actuación negativo, en lo que se traduce a un Dial negativo y esto no es posible.

Una alternativa para $\mathrm{S} 1$ es definir el Dial más bajo y no se configura como respaldo de ningún relé, pero no es posible calcular la corriente instantánea.

Este mismo problema se tiene cuando únicamente el generador 2 está deshabilitado o fuera de servicio, pero sucede es en S2 la dificultad de calcular sus parámetros; debido a que, de igual forma la corriente de arranque asignada a S2 (411.4 A), es menor a la corriente de corto vista por el mismo (283.92 A), al igual que la corriente de operación (380.311 A). 


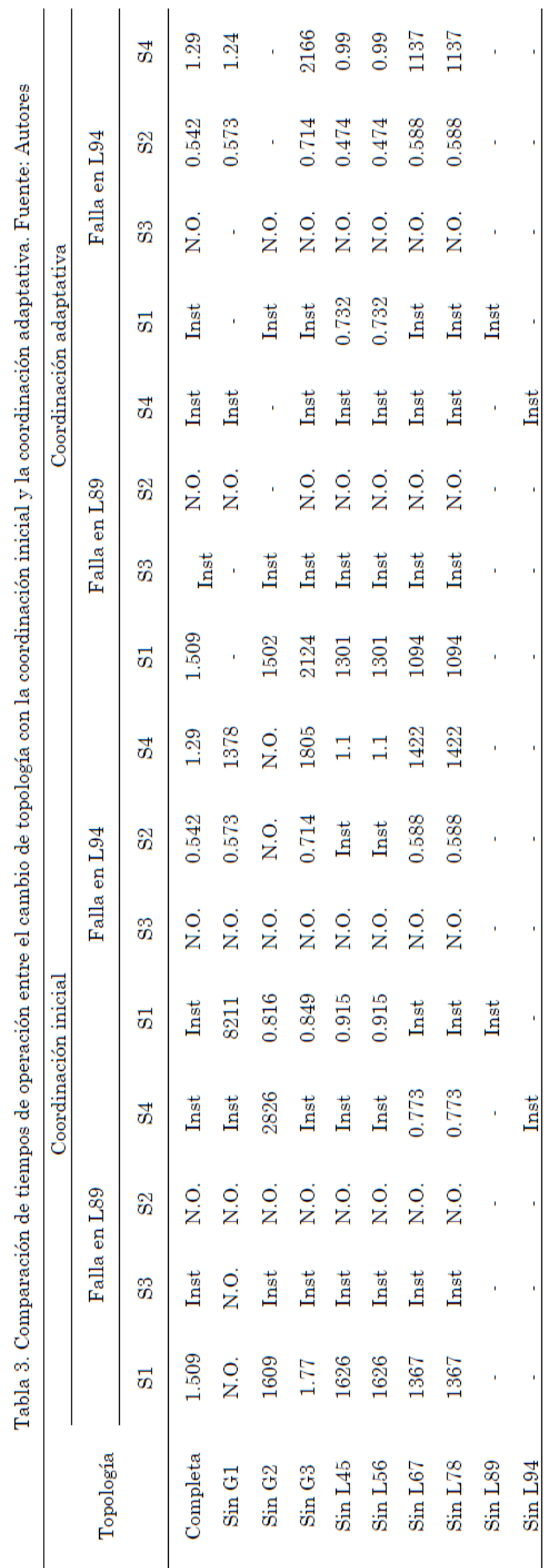

Estos dos casos se presentan por la modificación que sufre el sistema de potencia ante la pérdida de generación cuando el generador 1 o el generador 2 están fuera de servicio, ya que son los dos generadores más grandes y los que más potencia le entregan a la red.

En estos casos, los relés son desactivados temporalmente hasta que se presente un nuevo cambio de topología.

Cuando estos casos se presentan, los relés de la línea adyacente operan cuando existe una falla cerca a los relés desactivados. En la Fig. 10 se muestra el caso en que está deshabilitado el generador 1 y S4 opera instantáneamente en presencia de una falla trifásica al $50 \%$ de la línea 89.

Un caso similar ocurre en la Fig. 11 cuando únicamente sale el generador 2, donde no es posible calcular los parámetros de S2 y S4, y los relés S1 y S3 operan con el fin de aislar el sistema ante la falla trifásica.

En la Fig. 11a, la falla trifásica está al $50 \%$ de la línea 89 y el relé S3 opera instantáneamente cuando la detecta y S1 operaría en 1.502 segundos en el caso que S3 presente fallas de operación. En la Fig. $11 \mathrm{~b}$, la falla propuesta es localizada al 50\% de la línea 94, y en este caso únicamente el relé S1 opera $\mathrm{y}$ lo hace de forma instantánea, aislando el circuito de la falla.

Como puede observarse en los resultados expuestos anteriormente, existe un notable cambio en el tiempo de respuesta de las protecciones eléctricas, una vez se aplica el algoritmo adaptativo para cambios de topología en la red eléctrica.

Dicha implementación en el sector eléctrico colombiano generaría beneficios en la estabilidad de la red, reduciendo las salidas inesperadas de elementos en el sistema, generando mayor confiabilidad en los equipos de protección y asegurando una mejora en la operación del sistema. 

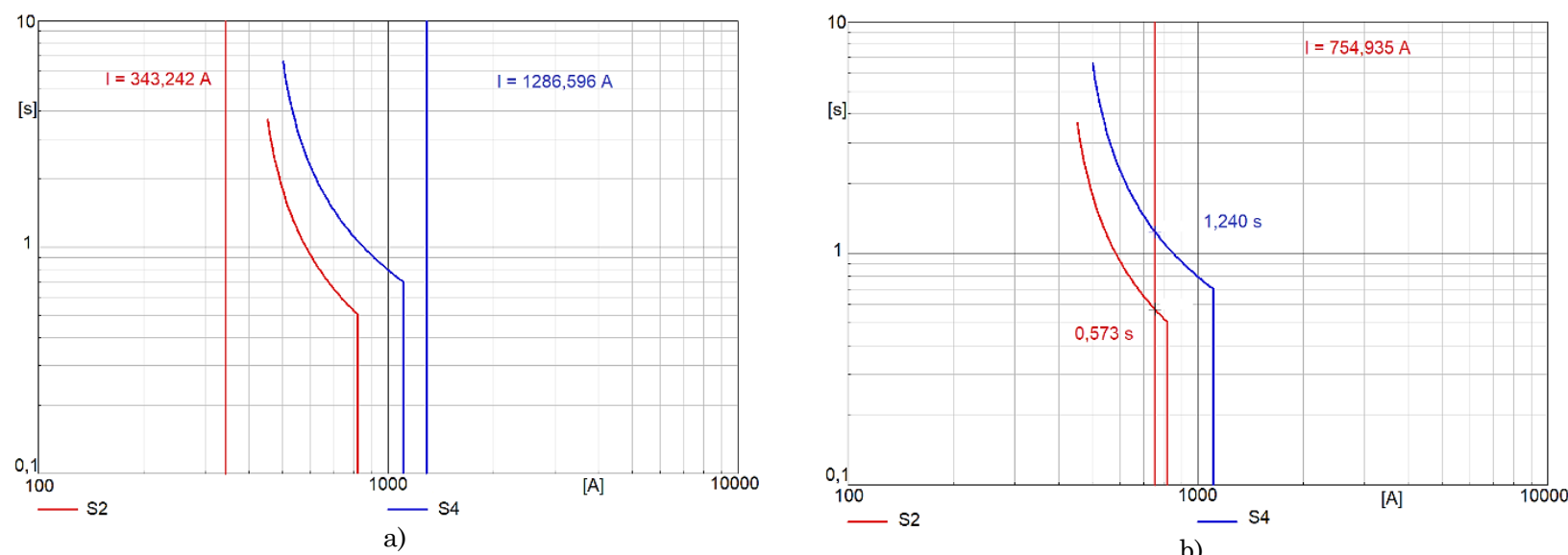

Fig. 10. Operación de relés S2 y S4. Generador 1 deshabilitado. a) Falla en línea 89. b) Falla en línea 94. Fuente: autores.

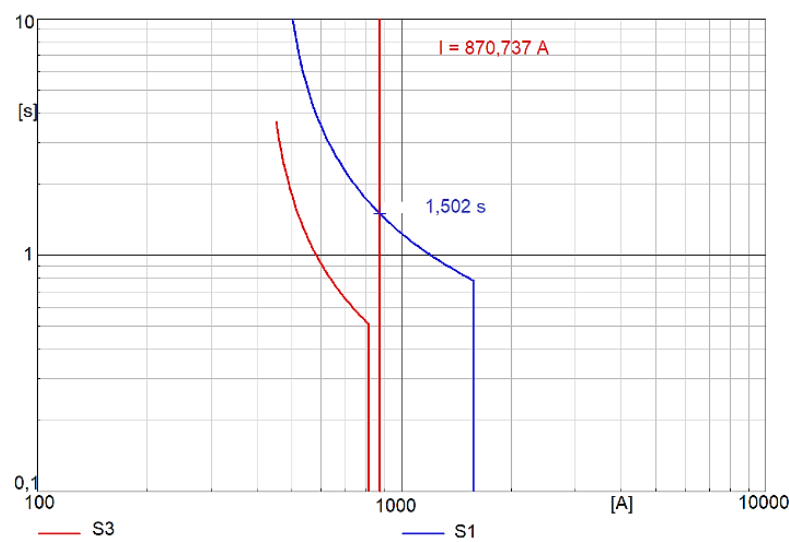

a)

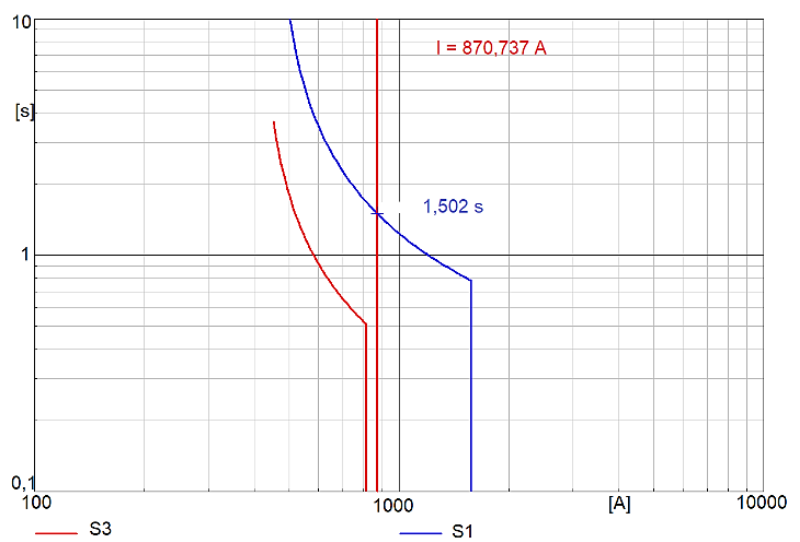

b)

Fig. 11. Operación de relés S1 y S3. Generador 2 deshabilitado. a) Falla en línea 89.b) Falla en línea 94. Fuente: autores.

Esto, en contraste con los esquemas de protección que se implementan actualmente en el país, los cuales no cuentan con la característica de modificar de manera automática los parámetros de operación a partir de la detección de cambios en la topología de la red eléctrica a la cual pertenecen.

\section{CONCLUSIONES}

La implementación de un algoritmo adaptativo de coordinación de protecciones resulta muy práctica en el momento que se presenten contingencias que ocasionen salidas inesperadas de elementos del sistema, manifestando un cambio de topología de la red eléctrica. El algoritmo detecta estos cambios y automáticamente ejecuta el cálculo de parámetros para los relés con base en la nueva topología.

Los resultados de implementar este algoritmo adaptativo en el caso de estudio IEEE9 con cuatro protecciones de sobrecorriente muestran que, ante variaciones de topología del sistema de potencia, el algoritmo cambia los parámetros de los relés de sobrecorriente para tratar de mantener selectividad entre estos y evitar en lo posible, errores de operación ante una falla tanto en las líneas de transmisión como en las barras.

Se pudo evidenciar una disminución en los tiempos de operación en todos los casos aplicando el algoritmo adaptativo respecto a la coordinación inicial, mejorando la respuesta de las protecciones. 
Existen 4 topologías del sistema en los que ciertos relés no obtuvieron parámetros al variar la topología, pero son casos en los que las corrientes de operación y de cortocircuito impiden su cálculo, es decir, el nivel de corto del sistema ante estas topologías no permite una adecuada coordinación. En estos casos los relés de las líneas adyacentes sirven como un respaldo para aislar las líneas de la falla, mostrando que el sistema sigue estando protegido aún en ausencia de relés operativos.

Este tipo de coordinación manual es utilizada por su facilidad para el cálculo rápido de los parámetros de los relés, especialmente si se tiene una red eléctrica de gran tamaño. Esta coordinación proporciona resultados adecuados y aceptables para la protección de las líneas del sistema gracias a su selectividad. No obstante, estos parámetros podrían mejorar si se utiliza alguna forma alterna para su cálculo, como métodos de optimización, algoritmos genéticos, redes neuronales, entre otros; pero se tendría que evaluar el tiempo de cómputo empleado para dicho fin, el cual tendría que verificarse si se desea implementar en tiempo real.

Tras la evaluación del proceso y los resultados obtenidos, se plantea la posibilidad de extrapolar esta prueba de coordinación adaptativa de protecciones, en un segmento de red de CODENSA, en el sector eléctrico colombiano; con el fin, de monitorear su comportamiento ante la dinámica real de la red eléctrica. De este modo, se considera necesario realizar estudios sobre protocolos de comunicación como el estándar IEC 61850, para la transferencia de información entre subestaciones eléctricas, que permitirá comunicar los cambios de topología en la red y la reconfiguración de los relés

\section{REFERENCIAS}

[1] A. J. Urdaneta, L. G. Perez, and H. Restrepo, "Optimal coordination of directional overcurrent relays considering dynamic changes in the network topology," IEEE Trans. Power Deliv., vol. 12, no. 4, pp. 14581464, Oct 1997. https://doi.org/10.1109/61.634161.

[2] M. Ojaghi, Z. Sudi, and J. Faiz, "Implementation of full adaptive technique to optimal coordination of overcurrent relays," IEEE Trans. Power Deliv., vol. 28, no. 1, pp. 235-244, Jan. 2013. https://doi.org/10.1109/TPWRD.2012.222148 3.

[3] A. Y. Abdelaziz, H. E. A. Talaat, A. I. Nosseir, and A. A. Hajjar, "An adaptive protection scheme for optimal coordination of overcurrent relays," Electr. Power Syst. Res., vol. 61, no. 1, pp. 1-9, Feb. 2002. https://doi.org/10.1016/S0378-7796(01)001766.

[4] B. Chattopadhyay, M. S. Sachdev, and T. S. Sidhu, "An on-line relay coordination algorithm for adaptive protection using linear programming technique," IEEE Trans. Power Deliv., vol. 11, no. 1, pp. 165-173, Jan.1996. https://doi.org/10.1109/61.484013.

[5] M. H. Hussain, S. R. A. Rahim, and I. Musirin, "Optimal overcurrent relay coordination: A review," Procedia Eng., vol. 53, pp. 332-336, March. 2013. https://doi.org/10.1016/j.proeng.2013.02.043.

[6] M. Farzinfar, M. Jazaeri, and F. Razavi, "A new approach for optimal coordination of distance and directional over-current relays using multiple embedded crossover PSO," Int. J. Electr. Power Energy Syst., vol. 61, pp. 620-628, Oct. 2014. https://doi.org/10.1016/j.ijepes.2014.04.001.

[7] F. Razavi, H. A. Abyaneh, M. Al-Dabbagh, R. Mohammadi, and H. Torkaman, "A new comprehensive genetic algorithm method for optimal overcurrent relays coordination," Electr. Power Syst. Res., vol. 78, no. 4, pp. 713-720, Apr. 2008. https://doi.org/10.1016/j.epsr.2007.05.013.

[8] T. S. Sidhu and B. K. Talukdar, "Topology detection for adaptive protection of distribution networks," in Energy Management and Power Delivery, 1995. Proceedings of EMPD '95., 1995 International Conference on, 1995, singapure pp. $445-450$.

[9] I. Abdulhadi, F. Coffele, A. Dyśko, C. Booth, and G. Burt, "Adaptive protection architecture for the smart grid," in IEEE PES Innovative Smart Grid Technologies 
Conference $\quad 2011$. https://doi.org/10.1109/ISGTEurope.2011.616 2781.

[10] F. Coffele, C. Booth, and A. Dyśko, "An Adaptive Overcurrent Protection Scheme for Distribution Networks," IEEE Trans. Power Deliv., vol. 30, no. 2, pp. 561-568, Feb. 2015. Manchester. https://doi.org/10.1109/TPWRD.2013.229487 9.

[11] S. A. Ahmadi, H. Karami, and B. Gharehpetian, "Comprehensive coordination of combined directional overcurrent and distance relays considering miscoordination reduction," Int. J. Electr. Power Energy Syst., vol. 92, pp. 42-52, Nov. 2017. https://doi.org/10.1016/j.ijepes.2017.04.008.

[12] B. Chattopadhyay, M. S. Sachdev, and T. S. Sidhu, "An on-line relay coordination algorithm for adaptive protection using linear programming technique," IEEE Trans. Power Deliv., vol. 11, no. 1, pp. 165-173, Jan. 1996. https://doi.org/10.1109/61.484013 